\title{
Erratum
}

\section{Randomized response techniques for complex survey designs}

Raghunath Arnab

Department of Statistics, University of Botswana, Private Bag UB 00705, Gaborone, Botswana; (e-mail: arnabr@mopipi.ub.bw)

\section{Erratum to: Statistical Papers 48: 131 - 141 (2006)}

Due to an unfortunate mistake, the authorship was wrongly displayed. Please recognize that Raghunath Arnab is the sole author of this article. 